Gazi University
Journal of Science
$\mathrm{http} / /$ dergipark.gov.tr/gujs

\title{
Alpha Power Odd Generalized Exponential Family of Distributions: Model, Properties and Applications
}

\author{
Ibrahim ELBATAL ${ }^{1}$ (D), Selen CAKMAKYAPAN ${ }^{2 *(D)}$, Gamze OZEL $^{3}$ \\ ${ }^{1}$ Department of Mathematics and Statistics, Imam Mohammad Ibn Saud Islamic University, 11432, Riyad, Saudi Arabia, \\ ${ }^{2}$ Department of Statistics, Istanbul Medeniyet University, 34700, Istanbul, Turkey, \\ ${ }^{3}$ Department of Statistics, Hacettepe University, 06800, Ankara, Turkey.
}

\section{Highlights}

- This paper focuses on a new distribution family.

- Three new distributions are defined from the new proposed distribution family.

- Applications based on real data sets are the importance and flexibility of the proposed models.

\section{Article Info}

Received: 26 Jan 2021 Accepted: 9 Oct 2021

Keywords

Alpha power

transformation

Odd generalized

exponential family,

Moments

Maximum likelihood

estimation

\begin{abstract}
In this paper, we exhibit a general family of distributions called alpha power odd generalized exponential family of distributions. The new family is very flexible with increasing, decreasing, J, reversed-J, bathtub shapes. Statistical properties of the family such as quantile, expansion of density function, moments, incomplete moments, mean deviation, Bonferroni and Lorenz curves are proposed. The method of maximum likelihood to estimate the model parameters is used. Three applications based on real data sets are the importance and flexibility of the three proposed models.
\end{abstract}

\section{INTRODUCTION}

The generation of new distributions beginning with a baseline distribution with parameters to existing distributions for building groups to illustrate flexibility is one of the favored research areas in the probability distribution submitted. In several applied fields, such as economics, environmental sciences, architecture, biological research, etc., many methods of applying a parameter to distributions have been proposed and used to model results. Some well-known families are the Marshall-Olkin-G by Marshall and Olkin [1], the beta-G by Eugene et al. [2], odd log-logistic-G by Gleaton and Lynch [3,4] the transmuted-G by Shaw and Buckley [5], the gamma-G by Zografos and Balakrishnan [6], the Kumaraswamy-G by Cordeiro and de Castro [7], the logistic-G by Torabi and Montazeri [8], exponentiated generalized-G by Cordeiro et al. [9], the McDonald-G by Alexander et al. [10], T-X family by Alzaatreh et al. [11], the Weibull-G by Bourguignon et al. [12], the generalized Lindley family by Cakmakyapan and Ozel [13], the odd generalized exponential (OGE) family by Tahir et al. [14], the beta odd log-logistic generalized by Cordeiro et al. [15], the generalized transmuted-G by Nofal et al. [16], alpha power transformated family of 
distributions introduced by Mahdavi and Kundu [17], alpha logarithmic transformation family of distributions introduced by Karakaya et al. [18] etc.

This article's purpose is to extract a new flexible distribution family, namely the distribution family of alpha power odd generalized exponential ( $\mathrm{AP}_{\mathrm{OGE}}$ ). The feature of the hazard rate will increase, decrease, $\mathrm{J}$, reverse $\mathrm{J}$, shaped bathtub and upside-down bathtub. In this case, the odd generalized exponential (OGE) family is called the baseline distribution $G$ for the transformed alpha power family and some of the new family's general properties are obtained.

The rest of the paper is organized as follows: In section 2, we present new alpha power odd-generalized exponential family $\left(\mathrm{AP}_{\mathrm{OGE}}\right)$ of distributions, and some special cases. Useful expansions for density and cumulative density functions of this family are derived in section 3. Statistical properties of AP OGE included quantile, ordinary, generating functions, conditional moments, mean deviation and Bonferroni and Lorenz curves are provided in section 4. Maximum likelihood estimation is performed in section 5. A simulation study is conducted to indicate performance for new family in section 6 . Then, applications are presented in section 7. The conclusion is given in section 8 .

\section{NEW FAMILY}

In this section we consider a cumulative distribution function (cdf) $G(x, \varphi)$ with the probability density function (pdf) $g(x, \varphi)$ and survival function $\bar{G}(x, \varphi)$ of a baseline model with parameter vector $\varphi$. Mahdavi and Kundu [17] proposed alpha power transformated family of distributions with the cdf and the pdf given by

$F_{A P}(x ; \alpha, \varphi)=\left\{\begin{array}{lr}\frac{\alpha^{G(x ; \varphi)}-1}{\alpha-1}, & \alpha>0, \alpha \neq 1, \quad x>0 \\ G(x ; \varphi), & \alpha=1, \quad x>0\end{array}\right.$

and

$f_{A P}(x ; \alpha, \varphi)=\left\{\begin{array}{lc}\frac{\log (\alpha) \alpha^{G(x ; \varphi)} g(x ; \varphi)}{\alpha-1}, & \alpha>0, \alpha \neq 1, \quad x>0 \\ g(x), & \alpha=1, \quad x>0\end{array}\right.$,

respectively. Here, $\varphi$ is the vector of parameter with baseline cdf $G(x ; \varphi)$. Tahir et al. [14] presented the OGE family of distributions with the cdf and

$H_{O G E}(x ; \lambda, \theta, \varphi)=\left(1-e^{-\lambda\left(\frac{G(x ; \varphi)}{\bar{G}(x ; \varphi)}\right)}\right)^{\theta}, \quad x>0$

and the pdf

$h_{O G E}(x ; \lambda, \theta, \varphi)=\frac{\lambda \theta g(x ; \varphi)}{\bar{G}^{2}(x ; \varphi)} e^{-\lambda\left(\frac{G(x ; \varphi)}{\bar{G}(x ; \varphi)}\right)}\left[1-e^{-\lambda\left(\frac{G(x ; \varphi)}{\bar{G}(x ; \varphi)}\right)}\right]^{\theta-1}, x>0$,

respectively. Here, $\lambda>0$ and $\theta>0$ are two additional parameters and $g(x ; \varphi), G(x ; \varphi)$ are the pdf and the cdf of any baseline distribution depending on a vector of parameter $\varphi$. Motivated from the alpha power transformation and OGE family, we construct a new generalized family by inserting (3) into (1). The cdf and pdf for new class AP oge family of distributions are 
$F_{A P_{O G E}}(x ; \alpha, \lambda, \theta, \varphi)=\left\{\begin{array}{lr}\frac{\alpha\left(1-e^{-\lambda\left(\frac{G(x ; \varphi)}{\bar{G}(x ; \varphi)}\right)}\right)^{\theta}}{\alpha-1}, & \alpha>0, \alpha \neq 1, \quad x>0 \\ \left(1-e^{-\lambda\left(\frac{G(x ; \varphi)}{\bar{G}(x ; \varphi)}\right)}\right)^{\theta}, & \alpha=1, \quad x>0\end{array}\right.$

and

$$
\begin{aligned}
& f_{A P_{O G E}}(x ; \alpha, \lambda, \theta, \varphi)=
\end{aligned}
$$

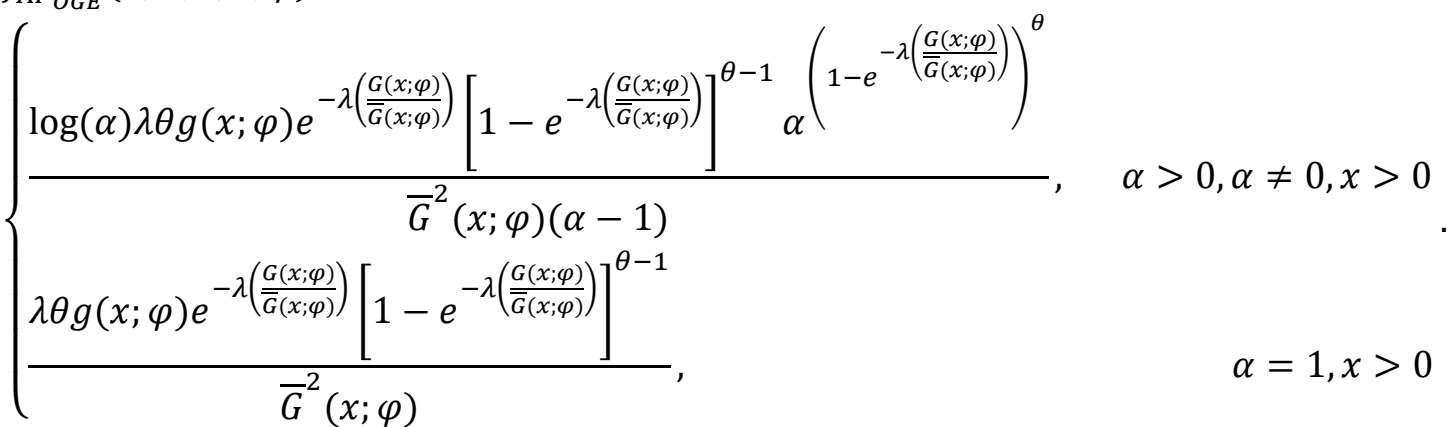

A random variable has the density function (6) is denoted by $X \sim A P_{O G E}(\eta)$ and $\eta=(\alpha, \lambda, \theta, \varphi)$. The hazard rate function (hrf) is given by

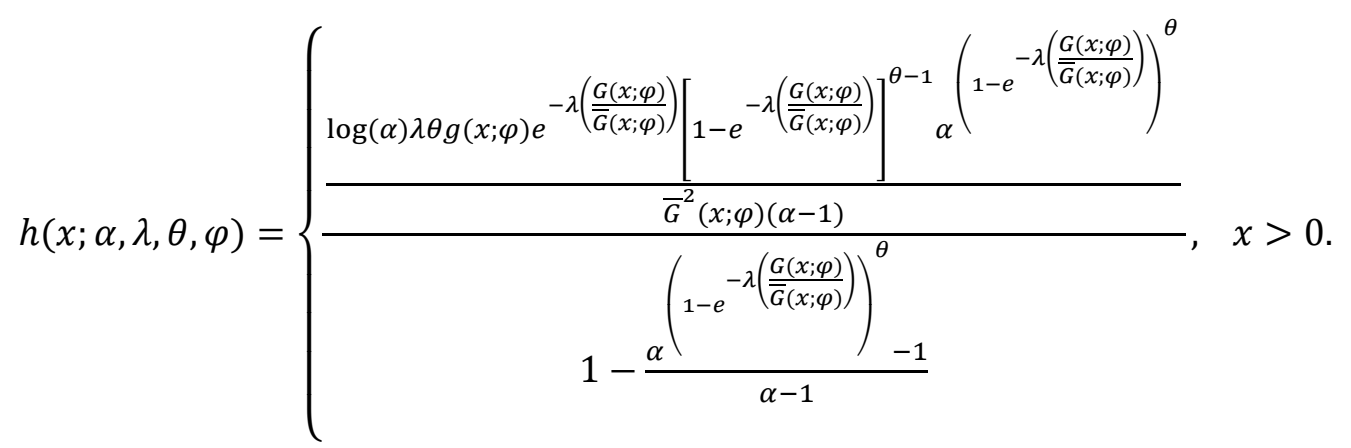

\subsection{Some Special Cases of the APoge-G Family}

In this subsection, we introduce special models for $\mathrm{AP}_{\mathrm{OGE}}$ family of distributions, "namely alpha power odd generalized exponential Lindley distribution, alpha power odd generalized exponential Rayleigh distribution and alpha power odd generalized exponential uniform distribution.

\subsubsection{Alpha power odd generalized exponential Lindley AP OGE $_{-L}$ distribution}

Consider the $G(x)=1-\left(1+\frac{a x}{1+a}\right) e^{-a x}$ and $g(x)=\frac{a^{2}}{1+a}(1+x) e^{-a x}$ of the Lindley distribution, then the cdf and pdf of the $A P_{O G E}-L$ model (for $x>0$ ) are

$F_{A P_{O G E}-L}(x ; \alpha, \lambda, \theta, a)=\left\{\begin{array}{rr}\frac{\alpha^{\left(1-e^{-\lambda\left(\left(1+\frac{a x}{1+a}\right)^{-1} e^{-a x}-1\right)}\right)^{\theta}}-1}{\alpha-1}, \quad \alpha>0, \alpha \neq 1, \quad x>0 \\ \left(1-e^{-\lambda\left(\left(1+\frac{a x}{1+a}\right)^{-1} e^{-a x}-1\right)}\right)^{\theta}, \quad \alpha=1, \quad x>0\end{array}\right.$

and 


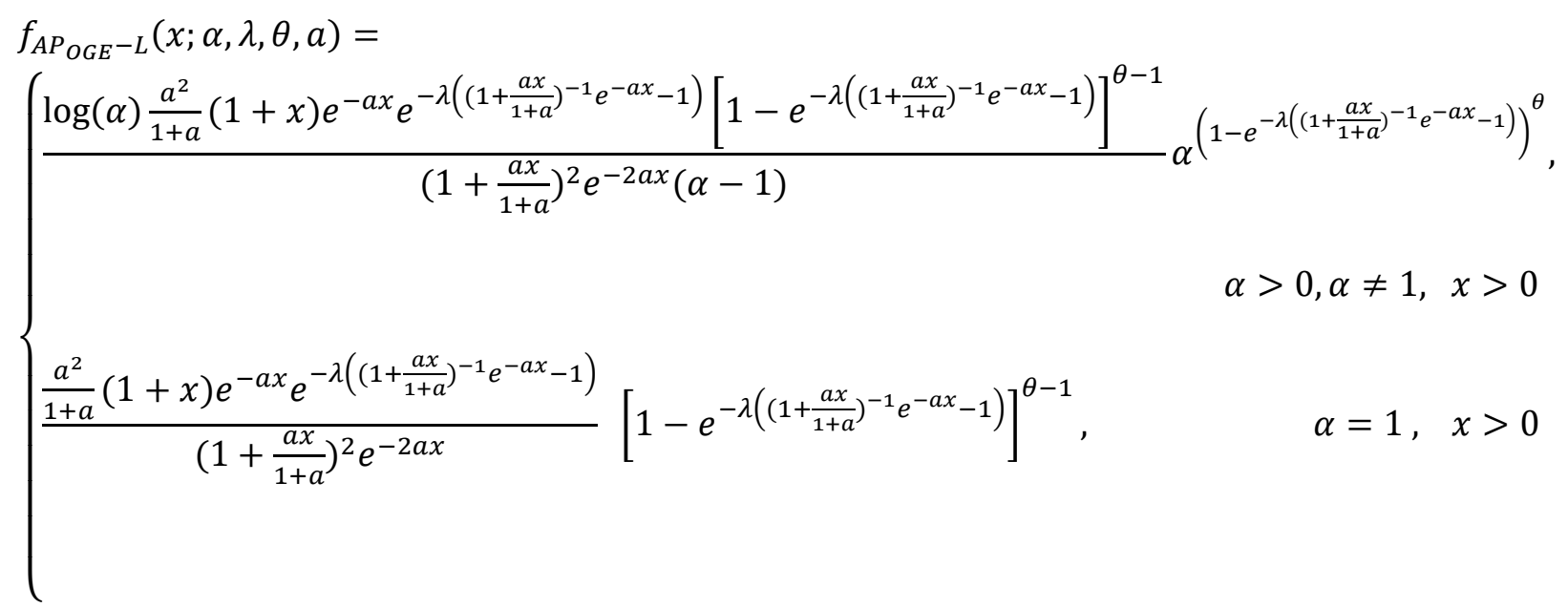

Figure 1 presents some hazard shapes of $A P_{O G E}-L$ distribution for arbitrary parameters. As seen in Figure 1 , the hrf should be increasing, decreasing and bathtube-shaped.
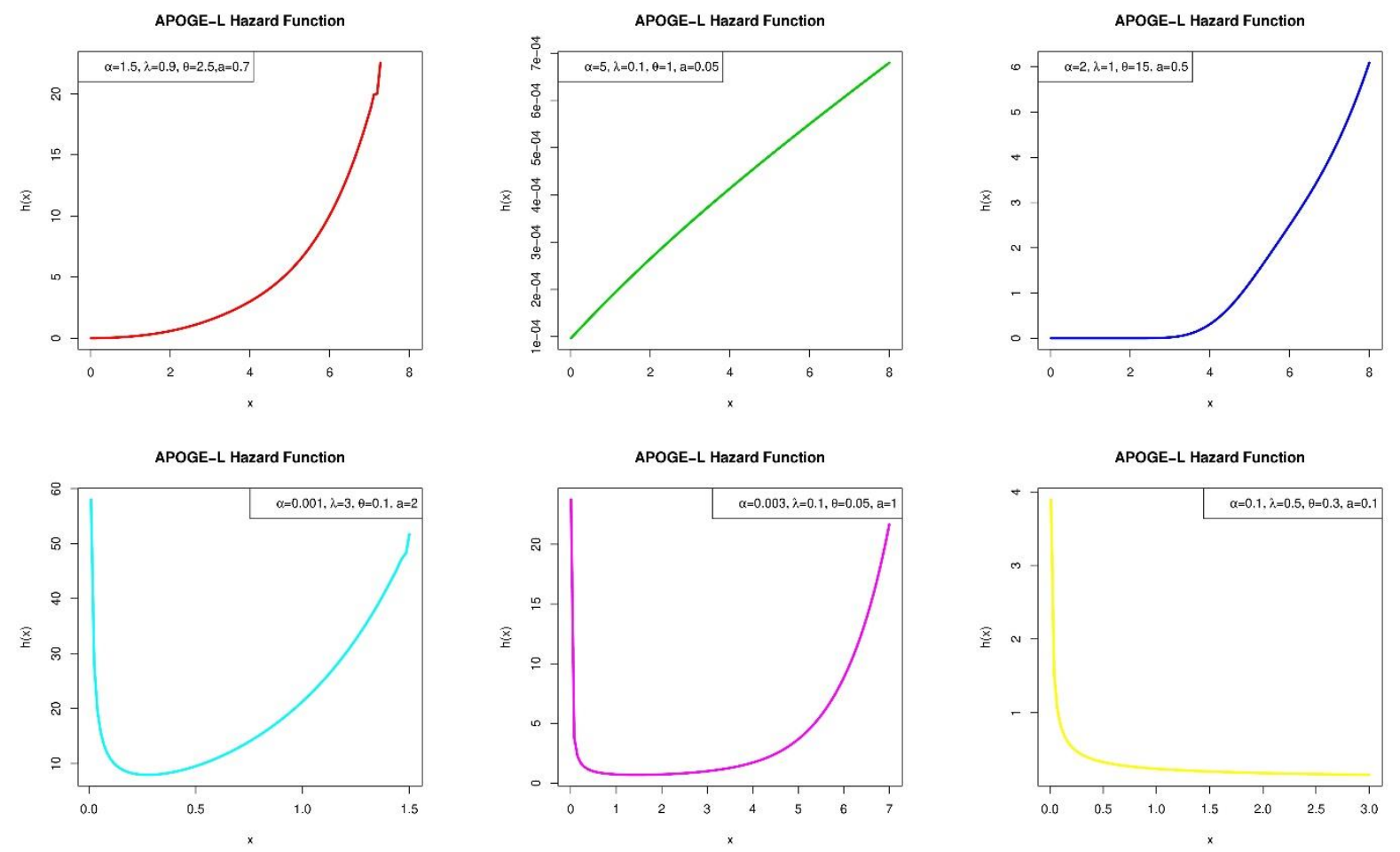

Figure 1. Some hazard shapes of $A P_{O G E}-L$ distribution for arbitrary parameters

\subsubsection{Alpha power odd generalized exponential Rayleigh $\left(A P_{O G E}-R\right)$ distribution}

Consider the cdf $G(x)=1-e^{-\frac{\mu}{2} x^{2}}$ and the pdf $g(x)=\mu x e^{-\frac{\mu}{2} x^{2}}$ of the Rayleigh distribution, then the cdf and pdf of the $A P_{O G E}-R$ model (for $x>0$ ) are 
$F_{A P_{O G E}-R}(x ; \alpha, \lambda, \theta, \mu)= \begin{cases}\frac{\alpha\left(1-e^{\lambda\left(e^{-\frac{\mu}{2} x^{2}}-1\right)}\right)^{\theta}}{\alpha-1}, & \alpha>0, \alpha \neq 1, x>0 \\ \left(1-e^{\lambda\left(e^{-\frac{\mu}{2} x^{2}}-1\right)}\right)^{\theta}, & \alpha=1, \quad x>0\end{cases}$

and

$$
\begin{aligned}
& f_{A P_{O G E^{-R}}}(x ; \alpha, \lambda, \theta, \mu)= \\
& \int \frac{\log (\alpha) \mu x e^{-\frac{\mu}{2} x^{2}} e^{-\lambda\left(e^{-\frac{\mu}{2} x^{2}}-1\right)}\left[1-e^{-\lambda\left(e^{-\frac{\mu}{2} x^{2}}-1\right)}\right]^{\theta-1}}{e^{-\mu x^{2}}(\alpha-1)} \alpha{ }^{\left(1-e^{-\lambda\left(e^{-\frac{\mu}{2} x^{2}}-1\right)}\right)^{\theta}}, \quad \alpha>0, \alpha \neq 1, \quad x>0 \\
& \frac{\mu x e^{-\frac{\mu}{2} x^{2}} e^{-\lambda\left(e^{-\frac{\mu}{2} x^{2}}-1\right)}\left[1-e^{-\lambda\left(e^{-\frac{\mu}{2} x^{2}}-1\right)}\right]^{\theta-1}}{e^{-\mu x^{2}}}, \\
& \alpha=1, \quad x>0
\end{aligned}
$$

We present the hrf of the $A P_{O G E}-R$ distribution for arbitrary parameters in Figure 2. The plots show that the hrf of $A P_{O G E}-R$ distribution should be increasing, decreasing, and bathtub shaped.
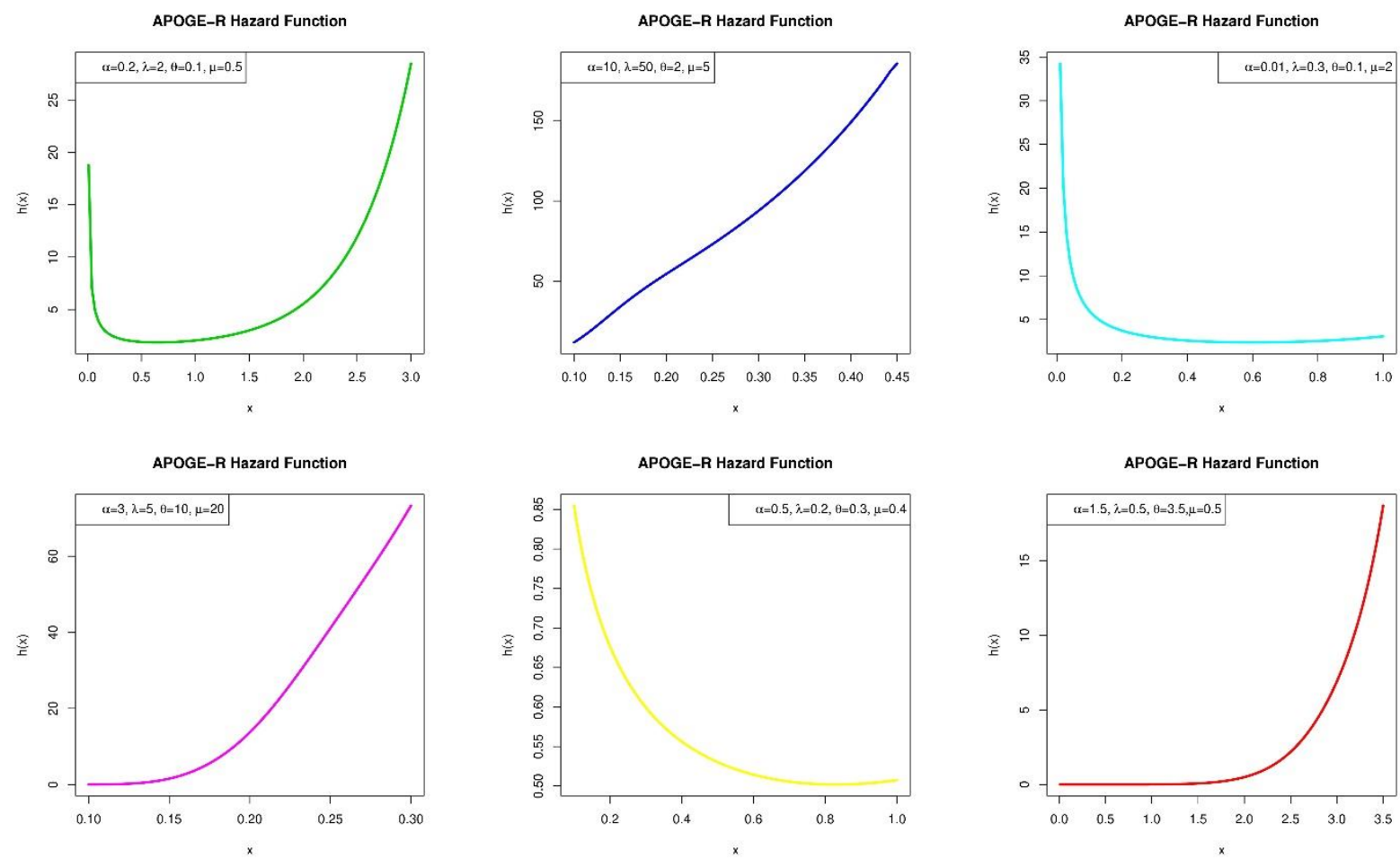

Figure 2. Some hazard shapes of $A P_{O G E}-R$ distribution for arbitrary parameters 


\subsubsection{Alpha power odd generalized exponential uniform $\left(A P_{O G E}-U\right)$ distribution}

Consider the uniform distribution on the interval $(0, \beta)$ with $G(x)=\frac{x}{\beta}$ and $g(x)=\frac{1}{\beta}$. The cdf and pdf for $A P_{O G E}-U$ distribution are, respectively, given by

$F_{A P_{O G E}-U}(x ; \alpha, \lambda, \theta, \beta)=\left\{\begin{array}{lr}\frac{\alpha\left(1-e^{-\lambda\left(\frac{x}{\beta}-1\right)}\right)^{\theta}-1}{\alpha-1}, & \alpha>0, \alpha \neq 0, x>0 \\ \left(1-e^{-\lambda\left(\frac{x}{\beta}-1\right)}\right)^{\theta}, & \alpha=1, x>0\end{array}\right.$

$f_{A P_{O G E}-U}(x ; \alpha, \lambda, \theta, \beta)= \begin{cases}\frac{\log (\alpha) \frac{\lambda \theta}{\beta} e^{-\lambda\left(\frac{x}{\beta}-1\right)}\left[1-e^{-\lambda\left(\frac{x}{\beta}-1\right)}\right]^{\theta-1} \alpha\left(1-e^{-\lambda\left(\frac{x}{\beta}-1\right)}\right)^{\theta}}{\left(1-\frac{x}{\beta}\right)^{2}(\alpha-1)}, \alpha>0, \alpha \neq 1, \quad x>0 \\ \frac{\frac{\lambda \theta}{\beta} e^{-\lambda\left(\frac{x}{\beta}-1\right)\left[1-e^{-\lambda\left(\frac{x}{\beta}-1\right)}\right]^{\theta-1}},}{\left(1-\frac{x}{\beta}\right)^{2}}, \alpha=1, \quad x>0\end{cases}$

The hrf for $A P_{O G E}-U$ distribution for several values of the parameters are given in Figure 3. The plots indicate that the hrf should be increasing, decreasing, J, reversed-J, bathtub shaped. 

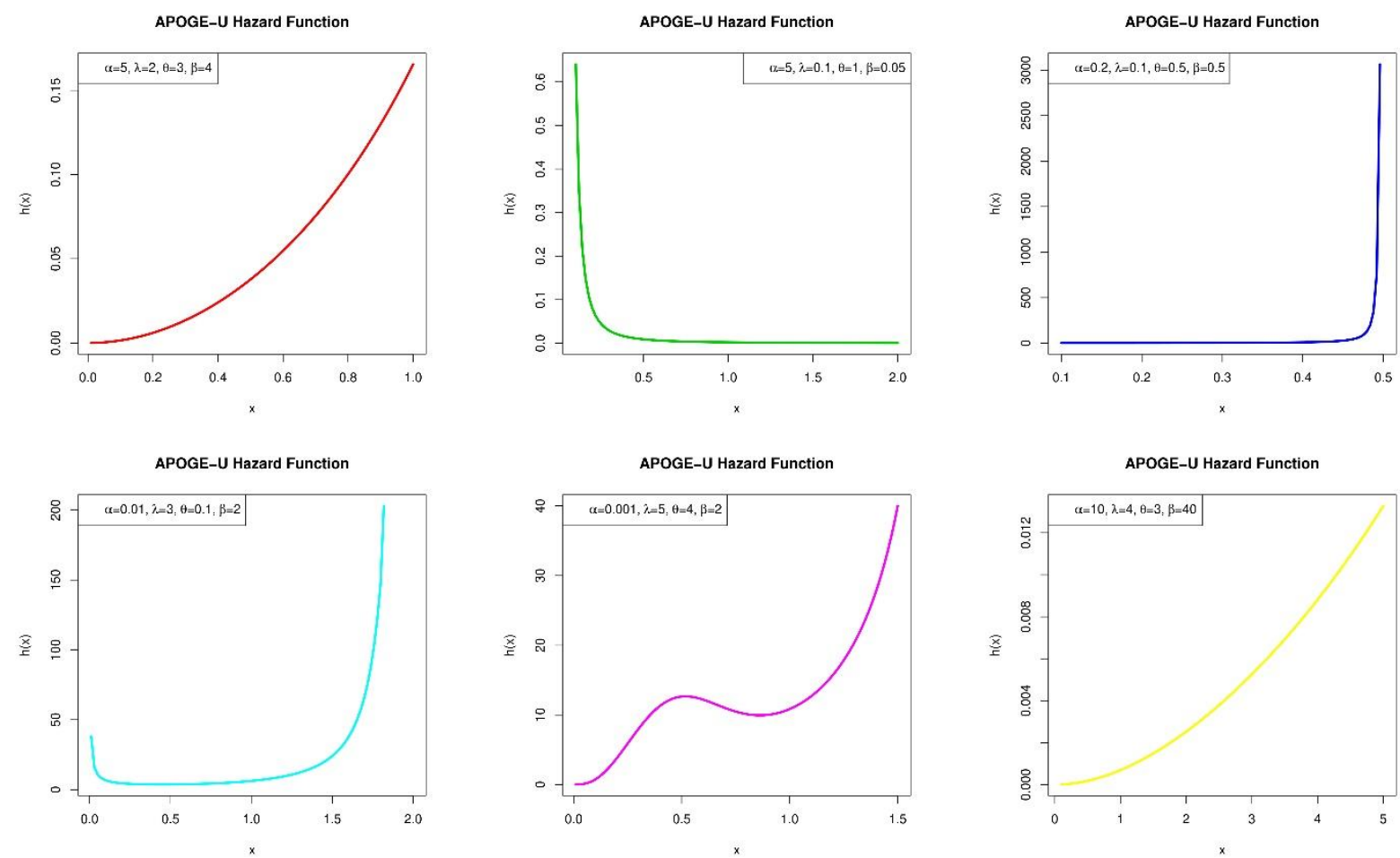

Figure 3. Some hazard shapes of $A P_{O G E}-U$ distribution for arbitrary parameters

\section{USEFUL EXPANSIONS}

We give a representation of the $A P_{O G E}$ family using the series representation

$\alpha^{Z}=\sum_{k=0}^{\infty} \frac{(\log \alpha)^{k}}{k !} Z^{k}$

and the binomial series expansion given by

$(1-z)^{b-1}=\sum_{k=0}^{\infty}(-1)^{j}\left(\begin{array}{l}b-1 \\ j\end{array}\right) z^{j}=\sum_{j=0}^{\infty}(-1)^{j} \frac{\Gamma(b)}{\Gamma(b-j) j !} z^{j}$.

Here, $b>0$ is the positive real non-integer and $|z|<1$. Density function for $A P_{O G E}$ family is expressed as

$f_{A P O G E}(x ; \eta)=\frac{\lambda \theta g(x ; \varphi)}{\bar{G}^{2}(x ; \varphi)} \sum_{i, j=0}^{\infty} \frac{(\log \alpha)^{i+1}}{i !(\alpha-1)}(-1)^{j}\left(\begin{array}{l}\theta(i+1)-1 \\ j\end{array}\right) e^{-\lambda(j+1)\left(\frac{G(x ; \varphi)}{\bar{G}(x ; \varphi)}\right)}$.

Expanding the term $e^{-\lambda(j+1)\left(\frac{G(x ; \varphi)}{\bar{G}(x ; \varphi)}\right)}$ in power series, we can write

$e^{-\lambda(j+1)\left(\frac{G(x ; \varphi)}{\bar{G}(x ; \varphi)}\right)}=\sum_{k=0}^{\infty} \frac{(-1)^{k} \lambda^{k}(j+1)^{k}}{k !} \frac{G(x ; \varphi)^{k}}{\bar{G}(x ; \varphi)^{k}}$.

Inserting (10) in (9), the $A P_{O G E}$ density can be written as

$f_{A P_{O G E}}(x ; \eta)=\sum_{i, j, k=0}^{\infty} \frac{\lambda \theta(\log \alpha)^{i+1}(-1)^{j+k} \lambda^{k}(j+1)^{k}}{(\alpha-1) i ! k !}\left(\begin{array}{l}\theta(i+1)-1 \\ j\end{array}\right) \times g(x ; \varphi) \frac{G(x ; \varphi)^{k}}{\overline{G(x ; \varphi)^{k+2}}}$.

Using the general binomial expansion to $(1-G(x ; \varphi))^{-(k+2)}$, we have 


$$
(1-G(x ; \varphi))^{-(k+2)}=\sum_{d=0}^{\infty} \frac{\Gamma(k+d+2)}{\Gamma(k+2) d !} G(x ; \varphi)^{d} .
$$

Applying the expansion in (12) to (11), $A P_{O G E}$ family is expressed as an infinite linear combination of the pdf for the exponentiated- $G$ (exp-G) family where

$f_{A P_{O G E}}(x ; \eta)=\sum_{k, d=0}^{\infty} \omega_{k, d}(k+d+1) g(x ; \varphi) G(x ; \varphi)^{k+d}=\sum_{k, d=0}^{\infty} \omega_{k, d} \pi_{(k+d+1)}(x ; \varphi)$.

Here, we define

$\omega_{k, d}=\sum_{i, j=0}^{\infty} \frac{\lambda \theta(\log \alpha)^{i+1}(-1)^{j+k} \lambda^{k}(j+1)^{k}}{i ! k !(k+d+1)(\alpha-1)}\left(\begin{array}{c}\theta(i+1)-1 \\ j\end{array}\right) \frac{\Gamma(k+d+2)}{\Gamma(k+2) d !}$

and $\pi_{v}(x)=v g(x) G(x)^{v-1}$ denotes the exp-G family with the parameter $v>0$. Equation (13) shows that density of $X$ is expressed as a linear mixture of exp- $G$ densities with power parameter $(k+d+1)$ [9]. Therefore, several statistical properties are obtained from exp- $G$ distribution. The cdf for $A P_{O G E}$ family is expressed as a mixture of the cdfs for exp- $G$. Then, we have

$F_{A P_{O G E}}(x ; \eta)=\sum_{k, d=0}^{\infty} \omega_{k, d} \Pi_{(k+d+1)}$.

\section{PROPERTIES}

\subsection{Quantile Function}

The $A P_{O G E}$ quantile function $x=Q(u)$ is obtained by inverting (5) as

$F^{-1}(u)=Q_{G}(u)=G^{-1}\left[\frac{\frac{-1}{\lambda} \log \left[1-\left(\frac{\log (1+u(\alpha-1))}{\log \alpha}\right)^{\frac{1}{\theta}}\right]}{1+\left\{\frac{-1}{\lambda} \log \left[1-\left(\frac{\log (1+u(\alpha-1))}{\log \alpha}\right) \frac{1}{\theta}\right]\right\}}\right]$.

We generate $X$ by taking $u$ as a uniform random variable in $(0,1)$. One of the earliest skewness measures to be suggested is the Bowley skewness [19] defined by

$S K=\frac{Q\left(\frac{3}{4}\right)+Q\left(\frac{1}{4}\right)-2 Q\left(\frac{1}{2}\right)}{Q\left(\frac{3}{4}\right)-Q\left(\frac{1}{4}\right)}$.

On the other hand, the Moors kurtosis [20] based on cotiles is given by

$K U=\frac{Q\left(\frac{7}{8}\right)-Q\left(\frac{5}{8}\right)+Q\left(\frac{3}{8}\right)-Q\left(\frac{1}{8}\right)}{Q\left(\frac{6}{8}\right)-Q\left(\frac{2}{8}\right)}$,

where $Q($.$) is the quantile function. S K$ and $K U$ are less sensitive to outliers. Positive kurtosis indicates heavy tails and peakness in comparison to the normal distribution for symmetric unimodal distributions, whereas negative kurtosis indicates light tails and flatness. For the normal distribution, $S K=K U=0$. 


\subsection{Moments and Moment Generating Functions}

\subsubsection{Moments}

Let $Z_{(k+d+1)}$ be a random variable with pdf of Exp-G family which has power parameter $k+d+1$. $r_{t h}$ moment for $A P_{O G E}$ family of distributions can be obtained from (13)

$\mu_{r}^{\prime}=E\left(X^{r}\right)=\sum_{k, d=0}^{\infty} \omega_{k, d} E\left(Z_{(k+d+1)}^{r}\right)$

where $Z_{(k+d+1)}$ denotes exp-G family by power parameter $k+d+1$. Another formula of $r_{t h}$ moment follows from (13) as

$\mu_{r}^{\prime}=E\left(X^{r}\right)=\sum_{k, d=0}^{\infty} \omega_{k, d} E\left(Z_{(k+d+1)}^{r}\right)$

where

$E\left(Z_{v}^{r}\right)=v_{-\infty}^{\infty} x^{r} g(x ; \varphi) G(x ; \varphi)^{\nu-1}, \quad v>0$

is calculated via baseline quantile function $Q_{G}(u)=G^{-1}(u)$ as

$E\left(Z_{v}^{r}\right)=v_{0}^{1} u^{v-1} Q_{G}(u)^{r} d u$.

Now, we introduce two formulae for the moment generating function (mgf). The first formula can be calculated from Equation (13) as follows:

$M_{X}(t)=E\left(e^{t X}\right)=\sum_{k, d=0}^{\infty} \omega_{k, d} M_{k+d+1}(t)$,

where $M_{k+d+1}(t)$ is the mgf of $Z_{(k+d+1)}$. Consequently, we can be easily determined $M_{X}(t)$ from generating function for exp-G. The second formula of $M_{X}(t)$ follows from (13) as

$M_{X}(t)=E\left(e^{t X}\right)=\sum_{k, d=0}^{\infty} \omega_{k, d} M_{k+d+1}(t)$

where $M_{\kappa}(t)$ is the mgf of random variable $Z_{\kappa}$ given by

$M_{\kappa}(t)=\kappa_{-\infty}^{\infty} e^{t X} g(x ; \varphi) G(x ; \varphi)^{\kappa-1}=\kappa_{0}^{1} u^{\kappa-1} e^{t Q_{G}(u)} d u, \quad \kappa>0$

which is calculated from baseline quantile function $Q_{G}(u)=G^{-1}(u)$.

\subsubsection{Conditional Moments}

The $s_{t h}$ incomplete moments of $X$ defined by $\vartheta_{s}(t)$ for any real $s>0$ is expressed from (13) as

$\vartheta_{s}(t)=\int_{-\infty}^{t} x^{s} f(x) d x=_{k, d=0}^{\infty} \omega_{k, d_{-\infty}}^{t} x^{s} \vartheta_{s,(k+d+1)}(t) d x$

where

$\vartheta_{s, \varkappa}(t)=\int_{0}^{G(t)} u^{\varkappa-1} Q_{G}(u)^{s} d u$ 
and $\vartheta_{s, \varkappa}(t)$ can be evaluated numerically.

\subsubsection{Mean Deviation}

The first incomplete moment is important and it is useful to obtain mean deviation about the mean and about the median, where the mean deviations give important information about characteristic of population and also have been applied of income fields and property in economics. If $X$ has the $A P_{O G E}$ family of distributions. The mean deviations about mean $\mu=E(X)$ and mean deviations about median $M$ are defined by

$\delta_{1}(x)=E\left|X-\mu_{1}^{\prime}\right|=2 \mu_{1}^{\prime} F\left(\mu_{1}^{\prime}\right)-2 \vartheta_{1}\left(\mu_{1}^{\prime}\right)$

and

$\delta_{2}(x)=E|X-M|=\mu_{1}^{\prime}-2 \vartheta_{1}(M)$

respectively, where $\mu_{1}^{\prime}=E(X), M=\operatorname{median}(X)=Q\left(\frac{1}{2}\right)$ and $\vartheta_{1}(t)$ is the first complete moment given by (17) with $s=1$.

\subsection{Bonferroni and Lorenz Curves}

For a positive random variable $X$ and a given probability $p$, Bonferroni and Lorenz curves are given by

$B(p)=\frac{1}{p \mu_{1}^{\prime}} \vartheta_{1}(q)$

respectively, where $\mu_{1}^{\prime}=E(X)$, and $q=Q(p)$ is the quantile function of $X$ at $p$.

$L(p)=\frac{1}{\mu_{1}^{\prime}} \vartheta_{1}(q)$

\section{MAXIMUM LIKELIHOOD ESTIMATION}

In this section, we obtain the maximum likelihood estimates (MLEs) for parameters of $A P_{O G E}$ family. Let $x_{1}, \ldots, x_{n}$ be a random sample of size $n$ from the $A P_{O G E}$ family given by (6). Let $\eta=(\alpha, \lambda, \theta, \varphi)^{T}$ be $p \times 1$ vector of parameters. The log-likelihood function of $A P_{O G E}$ family is given by

$$
\begin{array}{r}
L_{n}=n \log (\log (\alpha))-n \log (\alpha-1)+n \log (\lambda)+n \log (\theta)+\sum_{i=1}^{n} \log g\left(x_{i} ; \varphi\right)-\lambda \sum_{i=1}^{n} w_{i}+(\theta \\
-1) \sum_{i=1}^{n} \log \left(1-e^{-\lambda w_{i}}\right)+\sum_{i=1}^{n}\left(1-e^{-\lambda w_{i}}\right)^{\theta} \log (\alpha)-2 \sum_{i=1}^{n} \log \bar{G}\left(x_{i} ; \varphi\right)
\end{array}
$$

where $w_{i}=\frac{G(x ; \varphi)}{\bar{G}(x ; \varphi)}$. The log-likelihood of Equation (22) is maximized by using SAS or R-language or by solving the nonlinear likelihood equations obtained by differentiating (22). The components of the score function $U_{n}(\eta)=\frac{\partial L_{n}}{\partial \eta}=\left[\frac{\partial L_{n}}{\partial \alpha}, \frac{\partial L_{n}}{\partial \lambda}, \frac{\partial L_{n}}{\partial \theta}, \frac{\partial L_{n}}{\partial \varphi}\right]^{T}$ are given by

$U_{n}(\alpha)=\frac{n}{\alpha \log (\alpha)}-\frac{n}{\alpha-1}+\frac{1}{\alpha} \sum_{i=1}^{n}\left(1-e^{-\lambda w_{i}}\right)^{\theta}$,

$U_{n}(\lambda)=\frac{n}{\lambda}-\sum_{i=1}^{n} w_{i}+(\theta-1) \sum_{i=1}^{n} \frac{w_{i} e^{-\lambda w_{i}}}{\left(1-e^{-\lambda w_{i}}\right)}+\theta \log (\alpha) \sum_{i=1}^{n} w_{i} e^{-\lambda w_{i}}\left(1-e^{-\lambda w_{i}}\right)^{\theta-1}$, 
and

$\frac{\partial L_{n}}{\partial \theta}=\frac{n}{\theta}+\sum_{i=1}^{n} \log \left(1-e^{-\lambda w_{i}}\right)+\log (\alpha) \sum_{i=1}^{n} \log \left(1-e^{-\lambda w_{i}}\right)\left(1-e^{-\lambda w_{i}}\right)^{\theta}$

and

$$
\begin{gathered}
\frac{\partial L_{n}}{\partial \varphi_{k}}=\sum_{i=1}^{n} \frac{g^{\prime}\left(x_{i} ; \varphi\right)}{g\left(x_{i} ; \varphi\right)}-\lambda \sum_{i=1}^{n} \frac{\partial w_{i}}{\partial \varphi_{k}}+(\theta-1) \sum_{i=1}^{n} \frac{\lambda e^{-\lambda w_{i}}}{\left(1-e^{-\lambda w_{i}}\right)} \frac{\partial w_{i}}{\partial \varphi_{k}}+\log (\alpha) \theta \sum_{i=1}^{n} \lambda e^{-\lambda w_{i}}(1 \\
\left.-e^{-\lambda w_{i}}\right)^{\theta-1} \frac{\partial w_{i}}{\partial \varphi_{k}}-2 \sum_{i=1}^{n} \frac{\bar{G}_{\varphi_{k}}^{\prime}(x ; \varphi)}{\bar{G}(x ; \varphi)}
\end{gathered}
$$

where $g^{\prime}\left(x_{i} ; \varphi\right)=\frac{\partial g\left(x_{i} ; \varphi\right)}{\partial \varphi_{k}}$ and $\bar{G}_{\varphi_{k}}^{\prime}(x ; \varphi)=\frac{\partial \bar{G}(x ; \varphi)}{\partial \varphi_{k}}$. The MLEs of $\eta$, say $\hat{\eta}$, is obtained by solving nonlinear system $U_{n}(\eta)=0$.

\section{SIMULATION}

In this section, we perform a Monte Carlo simulation study for verifying finite sample behavior of MLEs of the parameters of the related parameters. We use a package programme in statistical software R, named Adequacy Model. In addition, we have shown that parameters can be obtained with a simple package program although pdfs seems complex. Simulation results are obtained from 1000 Monte Carlo replications. We generated random numbers using quantile functions of $A P_{O G E}-U, A P_{O G E}-L$ and $A P_{O G E}-R$ distirbutions with arbitrary parameters. Tables 1 and 2 show simulation results of MSEs (mean square errors) and their biases for estimating different combinations of real model parameters. We obtain estimations for three different the sample sizes $n=30,100,500$ and 1000. The results indicate that the MSEs and biases are very small and decrease while the sample sizes increase as expected. 
Table 1. Simulation results of MSEs for different combinations of real model parameters

\begin{tabular}{|c|c|c|c|c|c|}
\hline \multirow{2}{*}{ Distribution } & \multirow{2}{*}{ Real Parameters } & \multicolumn{4}{|c|}{ n } \\
\hline & & 30 & 100 & 500 & 1000 \\
\hline \multirow{12}{*}{ 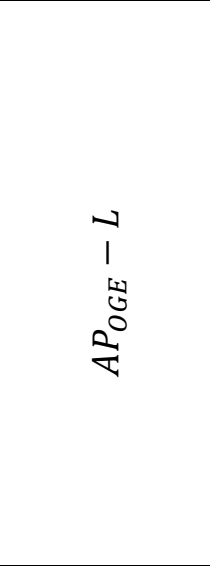 } & $\alpha=0.2$ & 0.025189 & 0.037011 & 0.003698 & 0.000281 \\
\hline & $\lambda=0.3$ & 0.022367 & 0.022140 & 0.008773 & 0.000015 \\
\hline & $\theta=0.4$ & 0.039744 & 0.017640 & 0.000889 & 0.000920 \\
\hline & $a=0.5$ & 0.016787 & 0.004739 & 0.001992 & 0.000968 \\
\hline & $\alpha=0.1$ & 0.033053 & 0.006444 & 0.000064 & 0.000027 \\
\hline & $\lambda=0.5$ & 0.009195 & 0.002429 & 0.000016 & 0.000009 \\
\hline & $\theta=0.2$ & 0.002030 & 0.000421 & 0.000009 & 0.000005 \\
\hline & $a=0.3$ & 0.011813 & 0.010506 & 0.000951 & 0.000095 \\
\hline & $\alpha=0.5$ & 0.019496 & 0.004753 & 0.001107 & 0.005587 \\
\hline & $\lambda=0.2$ & 0.034107 & 0.013732 & 0.002941 & 0.002407 \\
\hline & $\theta=0.7$ & 0.050224 & 0.016333 & 0.002492 & 0.001332 \\
\hline & $a=0.3$ & 0.011180 & 0.005164 & 0.000518 & 0.000339 \\
\hline \multirow{12}{*}{ 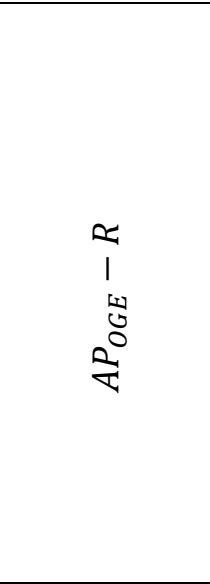 } & $\alpha=2.5$ & 0.109218 & 0.052775 & 0.026602 & 0.005181 \\
\hline & $\lambda=0.3$ & 0.192581 & 0.045081 & 0.008524 & 0.003723 \\
\hline & $\theta=0.4$ & 0.025768 & 0.005646 & 0.001097 & 0.000500 \\
\hline & $\mu=0.5$ & 0.065921 & 0.015989 & 0.003234 & 0.001519 \\
\hline & $\alpha=0.1$ & 0.049193 & 0.006204 & 0.000960 & 0.000417 \\
\hline & $\lambda=0.5$ & 0.056515 & 0.018375 & 0.001002 & 0.000404 \\
\hline & $\theta=0.2$ & 0.001637 & 0.000519 & 0.000111 & 0.000057 \\
\hline & $\mu=0.3$ & 0.160303 & 0.015602 & 0.001009 & 0.000414 \\
\hline & $\alpha=0.5$ & 0.021387 & 0.007730 & 0.001556 & 0.003073 \\
\hline & $\lambda=0.2$ & 0.034777 & 0.013587 & 0.002960 & 0.001648 \\
\hline & $\theta=0.7$ & 0.062698 & 0.017554 & 0.003248 & 0.001453 \\
\hline & $\mu=0.3$ & 0.017494 & 0.004527 & 0.000879 & 0.000432 \\
\hline \multirow{12}{*}{ 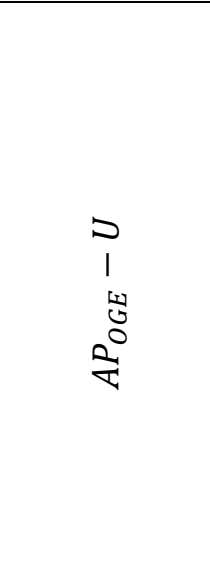 } & $\alpha=2.5$ & 0.035370 & 0.037013 & 0.000344 & 0.000064 \\
\hline & $\lambda=0.3$ & 0.036367 & 0.007290 & 0.000122 & 0.000061 \\
\hline & $\theta=0.4$ & 0.018456 & 0.003997 & 0.000068 & 0.000033 \\
\hline & $\beta=0.5$ & 0.000783 & 0.000147 & 0.000002 & 0.000001 \\
\hline & $\alpha=0.1$ & 0.019447 & 0.007586 & 0.000933 & 0.000040 \\
\hline & $\lambda=0.5$ & 0.106557 & 0.063994 & 0.008135 & 0.000244 \\
\hline & $\theta=0.2$ & 0.001520 & 0.000521 & 0.000104 & 0.000005 \\
\hline & $\beta=0.3$ & 0.010271 & 0.001916 & 0.000179 & 0.000006 \\
\hline & $\alpha=0.5$ & 0.003850 & 0.000088 & 0.000002 & 0.000000 \\
\hline & $\lambda=0.2$ & 0.008224 & 0.000258 & 0.000042 & 0.000016 \\
\hline & $\theta=0.7$ & 0.024025 & 0.000781 & 0.000115 & 0.000039 \\
\hline & $\beta=0.3$ & 0.000118 & 0.000028 & 0.000004 & 0.000000 \\
\hline
\end{tabular}


Table 2. Simulation results of biases for different combinations of real model parameters

\begin{tabular}{|c|c|c|c|c|c|}
\hline \multirow{2}{*}{ Distribution } & \multirow{2}{*}{ True Parameters } & \multicolumn{4}{|c|}{$\mathbf{n}$} \\
\hline & & 30 & 100 & 500 & 1000 \\
\hline \multirow{12}{*}{$\begin{array}{l}\overrightarrow{1} \\
\stackrel{5}{0} \\
\stackrel{0}{0} \\
\stackrel{0}{\sigma}\end{array}$} & $\alpha=2.5$ & -0.091028 & -0.027278 & -0.008180 & 0.004060 \\
\hline & $\lambda=0.3$ & -0.019731 & -0.032178 & -0.025291 & 0.009620 \\
\hline & $\theta=0.4$ & 0.065187 & 0.014504 & -0.009957 & 0.002382 \\
\hline & $a=0.5$ & 0.001539 & 0.017192 & 0.014969 & 0.003009 \\
\hline & $\alpha=0.1$ & 0.055664 & 0.020568 & -0.001749 & -0.002810 \\
\hline & $\lambda=0.5$ & -0.018288 & -0.004247 & -0.001111 & -0.001094 \\
\hline & $\theta=0.2$ & 0.001083 & -0.000422 & 0.002909 & 0.002409 \\
\hline & $a=0.3$ & 0.051784 & 0.017365 & 0.000949 & -0.000858 \\
\hline & $\alpha=0.5$ & -0.04704 & -0.005287 & -0.003992 & -0.003300 \\
\hline & $\lambda=0.2$ & 0.01138 & -0.020660 & 0.002399 & 0.003115 \\
\hline & $\theta=0.7$ & 0.03450 & -0.024322 & 0.000571 & 0.001102 \\
\hline & $a=0.3$ & 0.04363 & 0.030873 & 0.002327 & 0.001243 \\
\hline \multirow{12}{*}{$\begin{array}{l}1 \\
1 \\
51 \\
0 \\
0 \\
0\end{array}$} & $\alpha=2.5$ & -0.105635 & -0.055816 & -0.011895 & -0.002856 \\
\hline & $\lambda=0.3$ & 0.104285 & 0.033896 & 0.009330 & 0.003022 \\
\hline & $\theta=0.4$ & 0.034219 & 0.009924 & 0.002346 & 0.000965 \\
\hline & $\mu=0.5$ & 0.086713 & 0.026021 & 0.004650 & 0.003505 \\
\hline & $\alpha=0.1$ & 0.074180 & 0.018094 & 0.000780 & -0.001945 \\
\hline & $\lambda=0.5$ & -0.103943 & -0.042121 & -0.000883 & 0.000652 \\
\hline & $\theta=0.2$ & 0.003854 & 0.002590 & 0.002624 & 0.002552 \\
\hline & $\mu=0.3$ & 0.244807 & 0.059231 & 0.005339 & 0.001720 \\
\hline & $\alpha=0.5$ & -0.049061 & -0.024423 & -0.004273 & -0.001199 \\
\hline & $\lambda=0.2$ & 0.013277 & 0.014192 & -0.000280 & -0.005280 \\
\hline & $\theta=0.7$ & 0.048102 & 0.018736 & 0.000917 & -0.005601 \\
\hline & $\mu=0.3$ & 0.053661 & 0.014032 & 0.004566 & 0.003241 \\
\hline \multirow{12}{*}{ 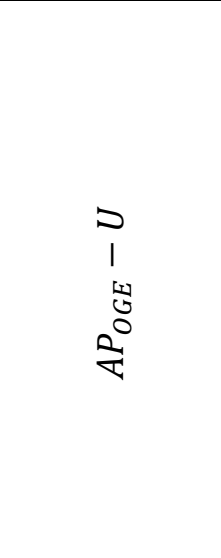 } & $\alpha=2.5$ & -0.034725 & -0.016541 & -0.004471 & -0.001156 \\
\hline & $\lambda=0.3$ & 0.014303 & -0.001337 & -0.004069 & -0.000262 \\
\hline & $\theta=0.4$ & 0.021489 & 0.005209 & -0.000309 & 0.000581 \\
\hline & $\beta=0.5$ & -0.006823 & -0.002354 & -0.000856 & -0.000306 \\
\hline & $\alpha=0.1$ & 0.010003 & -0.003987 & -0.003571 & -0.003363 \\
\hline & $\lambda=0.5$ & -0.244889 & -0.110825 & -0.023084 & -0.008070 \\
\hline & $\theta=0.2$ & 0.003217 & 0.004214 & 0.002755 & 0.002565 \\
\hline & $\beta=0.3$ & -0.080985 & -0.028905 & -0.005878 & -0.002396 \\
\hline & $\alpha=0.5$ & -0.020170 & -0.0073651 & -0.000746 & $-2.198 \mathrm{e}-04$ \\
\hline & $\lambda=0.2$ & -0.014190 & 0.0003659 & -0.001195 & $-8.768 \mathrm{e}-05$ \\
\hline & $\theta=0.7$ & 0.007113 & 0.0089225 & -0.000556 & $-1.322 \mathrm{e}-04$ \\
\hline & $\beta=0.3$ & -0.004796 & -0.0012513 & -0.000267 & $-1.673 e-04$ \\
\hline
\end{tabular}




\section{APPLICATIONS}

We demonstrate the utility of three real data sets for the $A P_{O G E}-G$ family, notably $A P_{O G E}-U, A P_{O G E}-$ $R$ and $A P_{O G E}-L$. The first data collection comprises 69 carbon fibers' tensile strength tests. They were measured at gauge lengths of $20 \mathrm{~mm}$ under strain as shown in Table 3 . The data set has already fitted other probability distributions by the authours [21-23]. We fit the data set also using $A P_{O G E}-U$ distribution. The results of the $A P_{O G E}-U$, Generalized Lindley (GL), Generalized Gamma (GG), Prakaamy (P), Sujatha (S), Ram Awadh (Ra), Pranav (PR), Ishita (I), Lindley (L), Exponential (E), weighted quasi Akash (WQA), three parameter weighted Lindley (TWL), Quasi Akash(QA) and Akash (A) distributions are given in Table 3. Table 3 contains MLEs and values for distribution statistics from AIC (Akaike information criterion), CAIC ( consistent AIC) and BIC (Bayesian information criterion). As seen in Table 4, $A P_{O G E}-U$ has the best fit to the data.

Table 3. Carbon Fibers Data

$1.312,1.314,1.479,1.552,1.700,1.803,1.861,1.865,1.944,1.958,1.966,1.997,006,2.021,2.027,2.055$, $2.063,2.098,2.140,2.179,2.224,2.240,2.253,2.270, .272,2.274,2.301,2.301,2.359,2.382,2.382,2.426$, $2.434,2.435,2.478,2.490, .511,2.514,2.535,2.554,2.566,2.570,2.586,2.629,2.633,2.642,2.648,2.684$, $.697,2.726,2.770,2.773,2.800,2.809,2.818,2.821,2.848,2.880,2.954,3.012, .067,3.084,3.090,3.096$, $3.128,3.233,3.433,3.585,3.585$

Table 4. MLEs and the values of AIC, CAIC, and BIC statistics

\begin{tabular}{|l|l|l|l|l|}
\hline Distribution & MLEs & AIC & CAIC & BIC \\
\hline$A P_{O G E}-U(\alpha, \lambda, \theta, \beta)$ & $10.495897,5.636075,13.438384,6.070391$ & 105.443 & 108.799 & 104.799 \\
\hline GL $(\alpha, \lambda, \theta)$ & $22.7198,4.7710,9.3907$ & 107.959 & 110.475 & 107.959 \\
\hline GG $(\alpha, \theta, \beta)$ & $3.586,12.6483,0.3044$ & 106,581 & 109.097 & 106.097 \\
\hline $\mathrm{P}(\alpha)$ & 2.0098 & 190.77 & 191.609 & 193.00 \\
\hline $\mathrm{S}(\lambda)$ & 0.936119 & 223.60 & 224.439 & 225.52 \\
\hline $\operatorname{Ra}(\beta)$ & 1.84921 & 209.41 & 210.249 & 209.60 \\
\hline $\operatorname{Pr}(\alpha)$ & 1.22514 & 219.12 & 219.959 & 221.03 \\
\hline $\mathrm{I}(a)$ & 0.931565 & 225.14 & 225.979 & 227.05 \\
\hline $\mathrm{L}(\theta)$ & 0.659000 & 240.38 & 241.219 & 242.61 \\
\hline $\mathrm{E}(\lambda)$ & 0.407941 & 263.74 & 264.579 & 265.97 \\
\hline WQA $(\alpha, \lambda, \beta)$ & $9.43570,7.2356,21.4177$ & 107.877 & 104.716 & 107.393 \\
\hline $\operatorname{TWL}(\lambda, \theta, \beta)$ & $9.35345,22.765,9.74956$ & 107.966 & 104.805 & 107.482 \\
\hline QAD $(\lambda, \theta)$ & $0.71313,59.325$ & 268.496 & 270.174 & 268.173 \\
\hline $\mathrm{A}(\theta)$ & 1.35544 & 165.727 & 166.566 & 165.565 \\
\hline
\end{tabular}

The second data collection reflects the relaxation periods of 20 analgesic patients in Table 5. Fayomi [24] suggested odd Frechet inverse Weibull (OFIW) distribution to better fit for the data set with AIC=44.253. On the other hand, APOGE-L distribution is more preferable than others with the smaller AIC value. The results for OFIW, new modified Weibull (NMW), generalized Sujatha (GS), S, Aradhana (Ar), L, E and Weibull (W) distributions are given in Table 6.

Table 5. Relief Times Data $1.1,1.4,1.3,1.7,1.9,1.8,1.6,2.2,1.7,2.7,4.1,1.8,1.5,1.2,1.4,3,1.7,2.3,1.6,2$

Table 6. MLEs and the values of AIC, CAIC, and BIC statistics

\begin{tabular}{|l|l|l|l|l|}
\hline Distribution & MLEs & AIC & CAIC & BIC \\
\hline$A P_{O G E}-L(\alpha, \lambda, \theta, a)$ & $0.067918,18.251489,8.215363,0.198803$ & 41.278 & 43.945 & 45.261 \\
\hline OFIW $(a, b, \alpha, \beta)$ & $0.208,25.815,13.215$ & 44.253 & 45.753 & 42.156 \\
\hline NMW $(\mu, \alpha, \lambda, \theta, a)$ & $0.1215,2.7837,0.000008227,0.0003,2.7871$ & 51.173 & 55.459 & 47.678 \\
\hline GS $(\alpha, \lambda)$ & $1.5712,222.235$ & 49.96 & 50.67 & 51.96 \\
\hline $\mathrm{S}(\lambda)$ & 11,367 & 59.49 & 59.71 & 60.49 \\
\hline
\end{tabular}




\begin{tabular}{|l|l|l|l|l|}
\hline $\operatorname{Ar}(\theta)$ & 11,232 & 58.37 & 58.59 & 59.36 \\
\hline $\mathrm{L}(\theta)$ & 0.8161 & 62.49 & 62.71 & 63.49 \\
\hline $\mathrm{E}(\lambda)$ & 0.5263 & 67.67 & 67.89 & 68.67 \\
\hline $\mathrm{W}(\alpha, \theta)$ & $2.7870,2.1300$ & 45.173 & 47.164 & 45.879 \\
\hline
\end{tabular}

The third data set covers the glass fibre strengths of $1.5 \mathrm{~cm}$ given in Table 7. Firstly, knowledge was collected by employees at the UK National Physical Laboratory. The data set is also very popular and it has been used many studies. Abdelfattah et al. [25] suggested a new distribution namely, Weibull generalized exponential distribution and fitted the data by the distribution. They obtained the best fit with AIC $=35.656$. We used $A P_{O G E}-R$ and suggest the distribution to fit with smaller AIC value given in Table 7.

Table 6. Glass Fibres Data

$0.55,0.93,1.25,1.36,1.49,1.52,1.58,1.61,1.64,1.68,1.73,1.81,2,0.74,1.04,1.27,1.39,1.49, .53$, $1.59,1.61,1.66,1.68,1.76,1.82,2.01,0.77,1.11,1.28,1.42,1.5,1.54,1.6,1.62,1.66,1.69, .76,1.84$, $2.24,0.81,1.13,1.29,1.48,1.5,1.55,1.61,1.62,1.66,1.7,1.77,1.84,0.84,1.24,1.3, .48,1.51,1.55$, $1.61,1.63,1.67,1.7,1.78,1.89$

Table 7. MLEs and the values of AIC, CAIC, and BIC statistics

\begin{tabular}{|l|l|l|l|l|}
\hline Distribution & MLEs & AIC & CAIC & BIC \\
\hline$A P_{O G E}-R(\alpha, \lambda, \theta, \mu)$ & $8.485560,0.873138,1.907908,0.951858$ & 34.651 & 35.340 & 43.223 \\
\hline $\mathrm{E}(\lambda)$ & 0.664 & 179.660 & 179.726 & 181.803 \\
\hline $\operatorname{GE}(\alpha, \lambda)$ & $2.6105,31.3032$ & 66.767 & 66.967 & 71.053 \\
\hline $\operatorname{BE~}(\alpha, \lambda, \theta)$ & $17.7786,22.7222,0.3898$ & 54.254 & 54.661 & 60.683 \\
\hline $\operatorname{BGE}(\alpha, \lambda, \theta, a)$ & $0.4125,93.4655,0.92271,22.6124$ & 39.199 & 39.889 & 47.772 \\
\hline WGE $(\lambda, \theta, a)$ & $56.881,4.893,0.222$ & 35.656 & 36.063 & 42.085 \\
\hline
\end{tabular}

Finally, we obtain graphs of density and cumulative density functions and histograms to see how better the distributions fitting the data sets in Figures 4 and 5. These figures show that the $A P_{O G E}$ family is can be used to model symmetric, left and right skewed data sets.

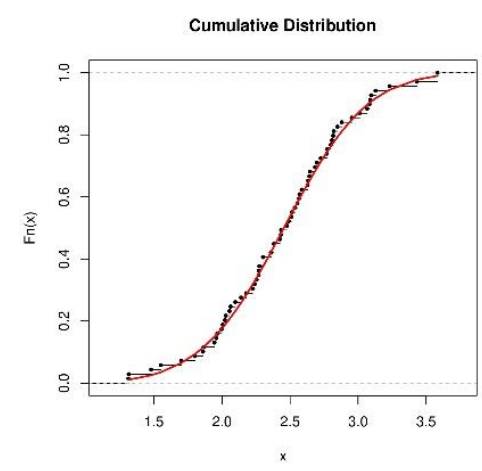

Carbon Fibres Data

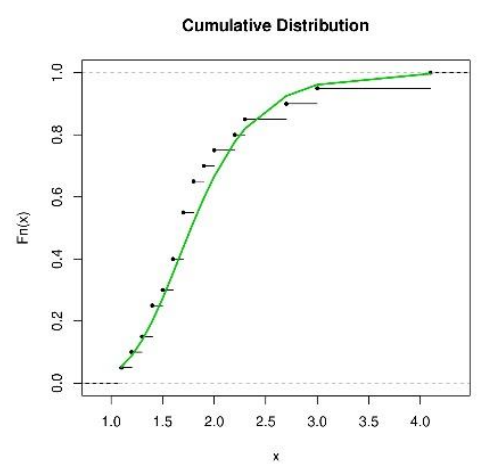

Relief Times Data

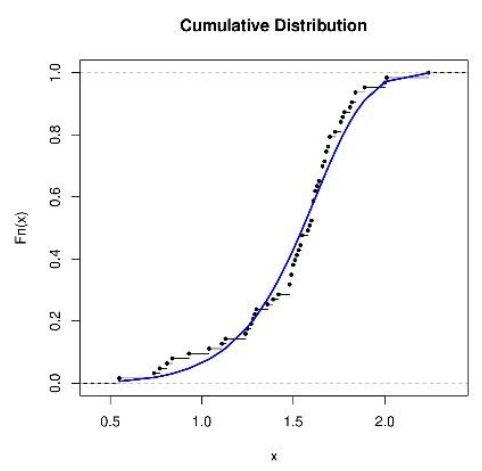

Glass Fibres Data

Figure 4. Graphs of emprical cdf and cdf for $A P_{O G E}-U, A P_{O G E}-R, A P_{O G E}-L$ 


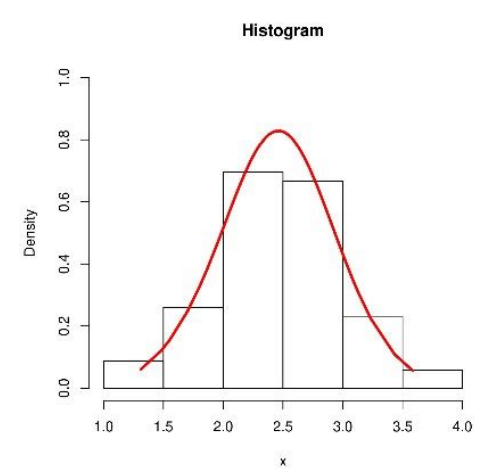

Carbon Fibres Data

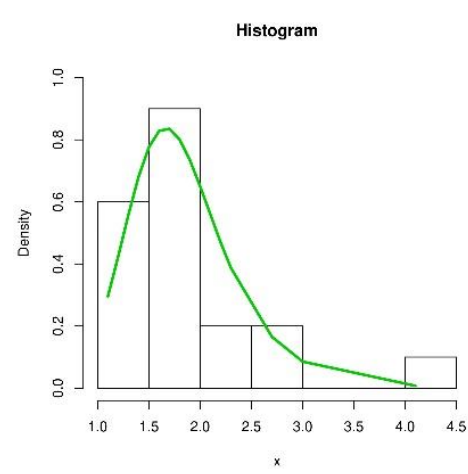

Relief Times Data

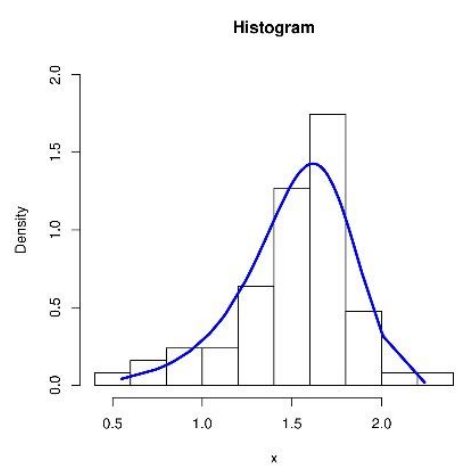

Glass Fibres Data

Figure 5. Histograms and fitted lines for $A P_{O G E}-U, A P_{O G E}-R, A P_{O G E}-L$

\section{CONCLUSION}

We propose a new family of distributions in this paper, called the alpha power odd generalized exponential distribution family. Several new family properties are discussed, including moments, moment generating function, quantile function, and special cases. The parameters are calculated using the maximum likelihood method. Then, the results of the Monte Carlo simulation show that the MLE outputs are very satisfactory. Applications show that, of all versions, the $A P_{O G E}$ family offers the best fit. The $A P_{O G E}$ family provides a closer fit to the histogram than the other models from plots for suited densities and histograms. The new family model can therefore be used in the study of data very efficiently. We hope that the proposed model can be efficiently used to fit real data as a competitive model.

\section{CONFLICTS OF INTEREST}

No conflict of interest was declared by the authors.

\section{REFERENCES}

[1] Marshall, A., and Olkin, I., "A new method for adding a parameter to a family of distributions with applications to the exponential and Weibull families", Biometrika, 84(3): 641- 652, (1997).

[2] Eugene, N., Lee, C., and Famoye, F., "Beta-normal distribution and its applications", Communication in Statistics- Theory and Methods, 31(4): 497-512, (2002).

[3] Gleaton, J. U., and Lynch, J. D., "On the distribution of the breaking strain of a bundle of brittle elastic bers", Advances in Applied Probability, 36: 98-115, (2004).

[4] Gleaton, J. U., and Lynch, J. D., "Properties of generalized log-logistic families of lifetime distributions", Journal of Probability and Statistics, 4: 51- 64, (2006).

[5] Shaw W.T., and Buckley I.R., "The Alchemy of probability distributions: beyond Gram-Charlier expansions, and a skew-kurtotic-normal distribution from a rank transmutation map", (2009). arXiv preprint arXiv:0901.0434.

[6] Zografos, K., and Balakrishnan, N., "On families of beta and generalized gamma-generated distributions and associated inference", Statistical Methodology, 6: 344 - 362, (2009).

[7] Cordeiro, G., and de Castro, M., "A new family of generalized distributions", Journal of Statistical Computation and Simulation, 81: 883- 898, (2011). 
[8] Torabi, H., and Montazeri, N. H., "The logistic-uniform distribution and its application", Communications in Statistics - Simulation and Computation, 43: 2551- 2569, (2014).

[9] Cordeiro, G., Ortega, E., and da Cunha, D.C., "The exponentiated generalized class of distributions", Data Science Journal, 11: 127, (2013).

[10] Alexander, C., Cordeiro, G. M., Ortega, E. M. M., and Sarabia, J. M., "Generalized betagenerated distributions", Computational Statistics \& Data Analysis, 56: 1880-1897, (2012).

[11] Alzaatreh, A., Lee, C., and Famoye, F., "A new method for generating families of continuous distributions", Metron, 71: 63-79, (2013).

[12] Bourguignon, M., Silva, R., and Cordeiro, G. M., "The Weibull-G family of probability distributions", Journal of Data Science, 12: 53-68, (2014).

[13] Cakmakyapan, S., and Ozel, G., "Generalized Lindley Family with application on Wind Speed Data", Pakistan Journal of Statistics and Operation Research, 387-397, (2021).

[14] Tahir, M. Cordeiro, G., Alizadeh, M., Mansoor, M., Zubair, M., and Hamedani, G., "The odd generalized exponential family of distributions with applications", Journal of Statistical Distributions and Applications, 2(1): 1-28, (2015).

[15] Cordeiro, G. M., Alizadeh, M., Tahir, H., Mansoor, M., Bourguignon, M., and Hamedani, G., "The beta odd log-logistic family of distributions", Hacettepe Journal of Mathematics and Statistics, 45(4): 1175-1202, (2016).

[16] Nofal, Z. M., Afy, A. Z., Yousof, H. M., and Cordeiro, G. M., The generalized transmuted-G family of distributions, Communication in Statistics- Theory and Methods, 46(8): 4119- 4136, (2017).

[17] Mahdavi A., and Kundu D., "A new method for generating distributions with an application to exponential distribution," Communication in Statistics- Theory and Methods, 46(13): 6543-6557, (2017).

[18] Karakaya, K., Kinaci, I., Coskun, K. U. S., and Yunus, A. K. D. O., "A new family of distributions", Hacettepe Journal of Mathematics and Statistics, 46(2), 303-314, (2017).

[19] Kenney, J., and Keeping, E., Mathematics of Statistics 1 th ed., D. Van Nostrand Company, Princeton, (1962).

[20] Moors, J.J.A., "A Quantile Alternative for Kurtosis", Journal of the Royal Statistical Society: Series D, 37(1): 25-32, (1988).

[21] Shanker, R., and Shukla, K., "On Modeling Of Lifetime Data Using Three-Parameter Generalized Lindley and Generalized Gamma Distributions", Biometrics \& Biostatistics International Journal, 4(7), (2016).

[22] Shukla, K.," A comparative study of one parameter lifetime distributions", Biometrics \& Biostatistics International Journal, 8(4): 111-123, (2019).

[23] Tesfalem, E., Shanker, R., Shukla, K.K., and Leonida, T.A., " Weighted quasi Akash distribution: properties and applications", American Journal of Mathematics and Statistics, 9(1): 30-43, (2019). 
[24] Fayomi, A., "The odd Frechet inverse Weibull distribution with application", Journal of Nonlinear Sciences and Applications, 12: 165-172, (2019).

[25] Abdelfattah, M., Beih, E.D., and Shamsan, A.G., "Weibull Generalized Exponential Distribution", (2016). arXiv:1606.07378v1 [math.ST]. 